\title{
Essais
}

Revue interdisciplinaire d'Humanités

$11 \mid 2017$

Fictions de l'identité

\section{Autriche, c'est-à-dire ce qui reste du mythe habsbourgeois}

Igor Fiatti

\section{(2) OpenEdition}

1 Journals

Édition électronique

URL : http://journals.openedition.org/essais/3314

DOI : 10.4000/essais.3314

ISSN : 2276-0970

Éditeur

École doctorale Montaigne Humanités

\section{Édition imprimée}

Date de publication : 15 mai 2017

Pagination : 39-52

ISBN : 979-10-97024-01-7

ISSN : 2417-4211

\section{Référence électronique}

Igor Fiatti, «Autriche, c'est-à-dire ce qui reste du mythe habsbourgeois », Essais [En ligne], 11 | 2017, mis en ligne le 14 octobre 2020, consulté le 22 octobre 2020. URL : http://journals.openedition.org/ essais/3314; DOI : https://doi.org/10.4000/essais.3314 


\section{Autriche, c'est-à-dire ce qui reste du mythe habsbourgeois}

\section{Igor Fiatti}

Est-ce qu'il existe une littérature autrichienne ? L'Autriche et sa production littéraire semblent condamnées à un désarroi existentiel, à tout jamais, à cause du poids spécifique (historico-culturel) de l'Allemagne et en raison de l'immanence du passé nazi sur la conscience collective. La réponse à la question posée, l'être ou ne pas être autrichien, est ainsi multiple, bigarrée et contradictoire, éphémère de sa nature. Et dans une telle recherche continuelle de lueur d'indices et de preuves identitaires, la résistance opposée aux engrenages de la puissante machine critico-éditoriale de l' "Anschluss tacite ${ }^{1}$ " est constituée, notamment par des fragments de mythe, des fossiles-conducteurs (H. Blumenberg) qui, mutatis mutandis, renvoient lucidement et désespérément aux sécurités de l'époque de l'ordo habsbourgeois.

Lorsqu'on fait face à un débat sur la littérature autrichienne, la diatribe austro-allemande se révèle une prémisse méthodologique aussi indispensable qu'inéluctable : la ligne de partage du flux dialectique distinguant catégoriquement les bassins du Rhin et du Danube se dessine tout d'abord, indépendamment du sujet et des thématiques traitées. Comme le note Claudio Magris, depuis la Chanson des Nibelungen, les deux fleuves se font face et se défient. Le Rhin c'est Siegfried, la virtus et la pureté germanique, la fidélité des Nibelungen, l'hérö̈sme chevaleresque et l'impavide fatalisme de l'âme allemande. Le Danube, par contre, c'est la Pannonie, le royaume d'Attila, c'est l'Orient, l'Asie qui déferle et détruit, à la fin de la Chanson des Nibelungen, la valeur germanique; quand les Burgondes le traversent, pour se rendre à la cour des perfides Huns, leur destin - un destin allemand - est scellé2. Les origines de ce conflit remontent à l'idée de Sprachnation, à la conception d'une litté-

1 Klaus Zeyringer, "Texte et contexte : la littérature autrichienne ", in Continuités et ruptures dans la littérature autrichienne : 17 essais, Nîmes, J. Chambon, 1996, p. 9-27, p. 10.

2 Claudio Magris, Danube (Danubio, 1986), trad. par Jean et Marie-Noëlle Pastureau, Paris, Gallimard, 1998, p. 37. 
rature (nationale) germanique uniformément basée sur un fond commun : la langue allemande. Toutefois, entre le XVIII et le XIX siècles, la langue et la littérature ont eu des fonctions et des rôles diamétralement opposés en Allemagne et en Autriche : d'un côté l'unité linguistique et le recours à une vision nationaliste de l'histoire littéraire devaient unir une structure fragmentée pour en faire un État national, de l'autre, on devait éviter le collapsus d'un Empire 3 . Une telle fusion entre langue et nation, systématique dans la conception de Herder et des frères Grimm, a empêché pour longtemps l'étude scientifique de la littérature autrichienne, en tant qu'entité séparée : par son critère deutsch-national, en Autriche la Geschichte der poetischen NationalLiteratur der Deutschen de Georg Gottfried Gervinus - publiée à partir du 1835 - a influencé non seulement les études de germanistique, mais aussi les réflexions sur l'identité de la production littéraire ${ }^{4}$. Vis-à-vis d'une pareille hégémonie, la première voix accréditée qui a cherché à revendiquer l'indépendance identitaire, ou au moins un distinguo, a été celle de Franz Grillparzer, le plus classique des écrivains autrichiens, incarnant l'essence de l'âme habsbourgeoise, son entière Stimmung humaine et culturelle ${ }^{5}$. En s'interrogeant sur les différences entre les auteurs allemands et leurs collègues autrichiens, Grillparzer a reconnu trois spécificités à ses compatriotes : la modestie, la saine raison et la vérité des sentiments ${ }^{6}$. C'est une tentative timide de différenciation, absolument dépourvue de toute rigueur scientifique, qui est dépassée en superficialité par les essais identitaires de Hugo von Hofmannsthal : cela est patent dans un tableau singulier intitulé Prussien et Autrichien ${ }^{7}$ (Preusse und Österreicher, 1917). Ici, il développe ce qui dans d'autres essais était implicite ou estompé : il oppose à l'Autrichien ce qu'il désigne par le mot Prussien, en soutenant que l'accomplissement de l'esprit allemand n'appartient pas à cette fausse généralisation qui est la Prusse, mais à l'Autriche supranationale.

3 Leslie Bodi, " Österreichische Literatur - Deutsche Literatur. Zur Frage von Literatur und nationaler Identität ", in Akten des VI. Internationalen Germanisten-Kongresses (Basel 1980), Bern/Frankfurt, Lang, p. 486-492. Cité par Klaus Zeyringer, "Texte et contexte : la littérature autrichienne ", in Continuités et ruptures dans la littérature autrichienne : 17 essais, op. cit., p. 12-13.

4 Klaus Zeyringer, "Texte et contexte : la littérature autrichienne ", in Continuités et ruptures dans la littérature autrichienne: 17 essais, op. cit., p. 16.

5 Gerhart Baumann, F. Grillparzer, Sein Werk und das österreichische Wesen, Freiburg/Wien, 1954, p. 4. Cité par Claudio Magris, Il mito absburgico nella letteratura austriaca (1963), Torino, Einaudi, 1996, p. 110.

6 Franz Grillparzer, «Worin unterscheiden sich sie die österreichischen Dichter von den übrigen? » (1837), in Sämtliche Werke, Peter Frank et Karl Pöernbacher (Hg.), München, Carl Hanser, 1964, Bd. 3, p. 809-811, p. 809. Cité par Klaus Amann, Die Dichter und die Politik, Essays zur österreichischen Literatur nach 1918, Wien, Édition Falter/Deuticke, 1992, p. 9.

7 Ibid., p. 10. 
Précisément dans l'immanence de die Katastrophe - à savoir la chute de l'Empire austro-hongrois - Hofmannsthal lance ainsi le modèle Autriche, une " autre Autriche ${ }^{8}$ ", autre culturellement de l'alliée Allemagne ; une Autricheécoumène (idéalisée et idéalisante) de peuples danubiens ; Mitteleuropa Allemande-slave-magyare-romance opposée au Reich germanique qui, comme le remarque Thomas Mann dans l'essai Considérations d'un apolitique (Betrachtungen eines Unpolitischen, 1918), doit sauver la Kultur, la profondeur allemande de l'esprit, le culte de l'intériorité, de la menace de la Zivilisation. Hofmannsthal veut au contraire guérir la scission entre Kultur et Zivilisation, en mettant l'accent sur l'organicité, la confiance, le lien, la tradition, sur les valeurs transcendantales de l'individu, sur l'humanitas, sur l'urbanitas'. Un tel distinguo sur l'autre Autriche, autre culturellement, se révélera central après la Deuxième Guerre mondiale dans la notion de «nationalité culturelle». Il s'agit d'une notion - nettement différente de la " nationalité linguistique " formulée par Herder - qui est apparue lorsque la société autrichienne s'est satisfaite de la déclaration de Moscou de 1943, selon laquelle l'Autriche fut le premier pays libre victime du nazisme ; à ça il faut ajouter le poids de la déclaration de neutralité (1955) de Vienne, qui a contribué à renforcer le lien entre l'idée d'Autriche fondée sur la culture et l'idée d'une littérature autrichienne. Et ici, temporellement et dialectiquement, le travail de Magris intervient : il a identifié dans son mémoire (1963) «le mythe habsbourgeois » en tant qu'élément fondateur de la littérature autrichienne, « la sublimation d'une société concrète en un monde de légende, pittoresque, sûr et ordonné ${ }^{10}$ ». (Cette diagnose de désir d'évasion et de fuite de la réalité a connu des critiques et des louanges, des contestataires et des partisans, mais, de toute manière, elle reste toujours un sujet critique incontournable dans l'anamnèse de la littérature autrichienne - et de la production littéraire de l'espace centro-européen dans son ensemble.)

\section{L'extinction impossible}

Pendant ce millénaire le catholicisme et les Habsbourg ont eu un effet destructeur sur la tête de notre peuple, un effet meurtrier, comme nous le savons et comme nous le prouve tout ce que nous voyons en Autriche ${ }^{11}$.

Cette phrase du roman Extinction : un effondrement (Auslöschung: ein zerfall, 1986) pourrait être considérée comme le syntagme de l'œuvre de Thomas Bernhard (1931-1989), un auteur qui, en qualité d'ethnopsychologue, a sondé la société autrichienne en brisant l'image idyllique qu'elle

$8 \quad I d$.

9 Ibid., p. 16.

10 Claudio Magris, Le mythe et l'empire dans la littérature autrichienne moderne (Il mito absburgico nella letteratura austriaca, 1963), trad. par Jean et Marie-Noëlle Pastureau, Paris, Gallimard, 1991, p. 26.

11 Thomas Bernhard, Extinction : un effondrement (Auslöschung: ein Zerfall, 1986), trad. par Gilbert Lambrichs, Paris, Gallimard, 2009, p. 116-117. 
souhaiterait donner d'elle-même ${ }^{12}$. Peut-être, comme aucun autre littérateur autrichien, Bernhard a enfoncé sa plume dans la vision terrifiante d'une Autriche réduite à sa petitesse physique et politique dans laquelle l'Histoire a enterré ses propres crimes ${ }^{13}$. Par un ton de censeur implacable, ses écrits brisent régulièrement le tabou de la conscience collective, ils dénoncent la loi du silence qui règne dans son pays et, avec véhémence, reprochent aux compatriotes le devoir de la mémoire ${ }^{14}$. Bernhard a publié Extinction en 1986, lorsque, entre lacérations et polémiques, à Vienne on élisait Kurt Waldheim Président de la République, découvrant en même temps son passé d'officier dans les rangs de la Wehrmacht. Trois ans après, Bernhard mourait à la fin d'une maladie longue et douloureuse, qui remontait à la tuberculose de sa jeunesse. Bien qu'il ne s'agisse pas de son dernier roman du point de vue de la rédaction, Extinction est le dernier roman publié du vivant de l'auteur - et aussi, pour cette raison, il a été lu comme son ultime testament poétique ${ }^{15}$.

Le protagoniste et le narrateur du livre, Franz Josef Murau, apprend par un télégramme la nouvelle de la mort de ses propres parents et de son frère aîné. De façon inattendue, Murau, qui conduit à Rome une forme d'existence artistico-philosophique, hérite ainsi d'un immense patrimoine concentré dans un château de l'Autriche supérieure, à Wolfsegg. La famille, la patrie, les origines, auxquelles il a toujours cherché à échapper, l'engloutissent dans une spirale exiguë et étouffante, dans un endroit physique et mental fait de conventions, utilités et bureaucratie, dans lequel l'Histoire du $\mathrm{XX}^{\mathrm{e}}$ siècle a menacé d'écraser inexorablement le « moi $^{16} »$. Murau représente son père comme un opportuniste compromis avec le nazisme, prisonnier d'une mentalité bureaucratique, obtuse ; le frère aîné comme un homme précocement tari, condamné à suivre les traces du père ; mais les mots les plus sévères sont réservés à la mère, quintessence de l'inculture, du monde de l'utilité et de l'argent. La mère, intéressée par la seule mondanité, amante d'un haut prélat romain, est un exemple de Puppenmutter mittel-européenne, une mère de poupées, une mère non plus naturelle qui ne met au monde que des enfants artificiels, Kunstkinder ${ }^{17}$; une Deutsche Frau, une nationale-socialiste hystérique ${ }^{18}$, capable de rendre méchant n’importe qui ${ }^{19}$.

12 Hélène Weishard, "De la malédiction d'être autrichien ", in Thomas Bernhard, Paris, Minerve, 2002, p. 121-139, p. 121.

13 Cf. Wendelin Schmidt-Dengler, "Thomas Bernhard, l'Autriche et l'Europe ", in Thomas Bernhard, op. cit., p. 143-150, p. 150.

14 Cf. Hélène Weishard, "De la malédiction d'être autrichien ", in Thomas Bernhard, op. cit., p. 121.

15 Luigi Reitani le remarque dans une recension du livre (L'Indice dei libri del mese, $\mathrm{n}^{\circ}$ 2, 1997), en renvoyant au volume de Hans Höller et Irene Heidelberg-Leonard, Antiautobiographie. Thomas Bernhards "Auslöschung", Frankfurt am Main, Suhrkamp, 1995.

16 Id.

17 Thomas Bernhard, Auslöschung: ein Zerfall, Frankfurt am Main, Suhrkamp, 1988, p. 124-25.

18 Ibid., p. 193.

19 Ibid., p. 298. 
Bernhard essaie de formuler la nature du nazisme autrichien qui, comme le note Hélène Weishard, serait le fruit d'une osmose entre le monde extérieur et "l'esprit autrichien » et permettrait à ses compatriotes de s'identifier à une idéologie totalitaire qui soutient le mal absolu. Toute aspiration démocratique, fondée sur la liberté et sur la tolérance, est évidemment bannie d'une telle vision ; c'est une caractéristique de l'homo austriacus qui lui fait dénier toute valeur à la vie ${ }^{20}$. Le nazi-catholicisme se révèle une disposition imprégnant la dimension publique et privée, une osmose ontologique que Murau - le protagoniste du livre - exemplifie, en soulignant que " cet État est comme ma famille, qui est taillée sur mesure pour la criminalité nationale-socialiste ${ }^{21}$ ".

Contre l'esprit national-socialiste-catholique de l'homme autrichien ${ }^{22}$, l' "Ungeist ${ }^{23}$ " autrichien, Bernhard-Murau étale sa propre volonté existentielle d'Extinction par un flux de conscience, aussi lourd que tourmenté, qui, dans l'écriture, se propose programmatiquement d'éteindre tout : la famille du protagoniste, son temps et Wolfsegg ${ }^{24}$, le " pervers ${ }^{25}$ " Wolfsegg, « infâme enfer provincial ${ }^{26}$ ". Sans chapitres ni paragraphes, ce roman est cadencé par une phrase qui devient obsédante à cause de sa réitération. Le narrateur-protagoniste répète maniaquement "Ich habe Gambetti gesagt " (j'ai dit à Gambetti), une formule auto-certifiant, qui se réfère à l'élève auquel il enseigne l'allemand. À l'instar des formules fixes qui ouvrent et ferment les récitations des communautés " sauvages ", comme le souligne Jean-François Lyotard avec le cas de Cashinahuas, la phrase, en légitimant le destinateur que présente son univers, se légitime elle-même auprès du destinataire. Le narrateur puise l'autorité pour raconter ses histoires dans son nom même. Mais son nom est autorisé par ses histoires, en particulier celles qui racontent la genèse des noms. "L'identification y règne en maîtresse ", "mutatis mutandis, l'autoidentification d'une culture passe par ce dispositif ${ }^{27} »$. La légitimité est donc assurée par la puissance du dispositif narratif : il couvre la multiplicité des familles de phrases et des genres de discours possibles, il enveloppe tous les noms ; il est toujours actualisable ; diachronique et parachronique, il assure la maîtrise du temps - c'est-à-dire de la vie et de la mort. Bref : le récit est l'autorité elle-même, il autorise un nous infrangible au-dehors duquel il n'y a que des ils. Ainsi l'organisation proposée par Bernhard est tout à fait opposée à celle cosmopolite des grands récits de légitimation qui caracté-

20 Hélène Weishard, «De la malédiction d'être autrichien ", in Thomas Bernhard, op. cit., p. 124.

21 Thomas Bernhard, Extinction : un effondrement, op. cit., p. 361.

22 Thomas Bernhard, Auslöschung : ein Zerfall, op. cit., p. 292.

23 Ibid., p. 293.

24 Ibid., p. 201.

25 Ibid., p. 442.

26 Thomas Bernhard, Extinction : un effondrement, op. cit., p. 233.

27 Jean-François Lyotard, Le postmoderne expliqué aux enfants, Paris, Galilée, 1988, p. 51-52. 
risent la modernité occidentale : elle ne concerne pas le "dépassement " de l'identité culturelle particulière vers une identité civique universelle ; rien dans la communauté brossée par Bernhard ne la conduit à se dialectiser vers une société de citoyen ${ }^{28}$. L'esthétique bernhardienne se révèle une esthétique moderne du sublime - moderne en tant que nostalgique, non adressée à la novatio postmoderne (Lyotard) ${ }^{29}$ - et elle s'ancre dans Wolfsegg, où, alors que le monde se retrouve dans un état chaotique, règne encore la "Ordnung ${ }^{30}$ ".

La poétique de Bernhard, même en blâmant in toto l'austriacité et son essence, n'arrive pas à faire abstraction de la nostalgie pour les sécurités de l'univers habsbourgeois. Si d'un côté Extinction est une dénonciation testamentaire de l'" effet destructeur " d'un millénaire sous le signe du catholicisme et des Habsbourg - qui a "éliminé la pensée dans notre peuple et fait fleurir la musique comme le plus inoffensif de tous les arts ${ }^{31}$, " affirmant la vision baroque d'un monde vu comme une manifestation de la plénitude et de la grâce divines que l'art devait matérialiser et glorifier ${ }^{32}$ - de l'autre "le nihilisme thérapeutique ${ }^{33}$ " de l'auteur montre des évidentes traces du mythe littéraire de l'Autriche-Hongrie. Bernhard, qui se limite à diagnostiquer la maladie d'une société, sans toutefois en proposer aucune cure, cède par exemple au paysage habsbourgeois : dans la tâche d'éteindre Wolfsegg par l'écriture ${ }^{34}$, les Préalpes autrichiennes se révèlent le paysage "le plus agréable et le plus apaisant ${ }^{35}$ ». C'est l'idylle de la Dorfgeschichte (le récit villageois) immortalisée au XIX ${ }^{\mathrm{e}}$ siècle par Peter Rosegger, et surtout par Adalbert Stifter, s'amplifiant au travers de l'écho de l'ode à ses habitants ${ }^{36}$, qui, par les idéaux éducatifs sains et agrestes, ont étayé la typique physionomie moralisante du "Strapaese ${ }^{37}$ " habsbourgeois et de sa littérature.

De toute manière, il est évident que, par rapport à l'Austria Felix peinte par ses prédécesseurs, Bernhard dessine un pays sans mémoire, incapable de retenir les leçons du passé : une Austria perennis, toujours semblable à

28 Cf. id.

29 François Lyotard, Le postmoderne expliqué aux enfants, op. cit., p. 26.

30 Thomas Bernhard, Auslöschung: ein Zerfall, op. cit, p. 369.

31 Thomas Bernhard, Extinction : un effondrement, op. cit., p. 117.

32 Cf. Carl Schorske, "Les deux cultures autrichiennes et leur destin moderne ", in Revue d'esthétique, $\mathrm{n}^{\circ}$ 9, 1985, p. 7-18.

33 Hélène Weishard, «De la malédiction d'être autrichien », in Thomas Bernhard, op. cit., p. 128.

34 Thomas Bernhard, Auslöschung: ein Zerfall, op. cit., p. 199.

35 Thomas Bernhard, Extinction: un effondrement, op. cit., p. 247.

36 Thomas Bernhard, Auslöschung: ein Zerfall, op. cit., p. 313.

37 Magris a intitulé "Strapaese absburgico " la quatrième partie de Il mito absburgico : comme le notent les traducteurs de la version française du livre, au lendemain de la Première Guerre mondiale le Strapaese désignait un courant littéraire tourné vers l'authenticité de la tradition paysanne et régionale italienne, en opposition à l'admiration pour les modèles étrangers ; attitude voisine du " racinement " proposé vingt ans auparavant en France par Maurice Barrès. (Claudio Magris, Le mythe et l'empire dans la littérature autrichienne moderne, op. cit., note 1 p. 389). 
elle-même ${ }^{38}$. Mais s'il est vrai que, à l'instar de Karl Kraus, il n'admet aucun compromis et transforme les loci amoeni en loci horribiles ${ }^{39}$, il est aussi vrai qu'une telle transfiguration n'est pas du tout complète : conscient du fait qu' en nature rien ne se perd, rien ne se crée, il applique la loi de Lavoisier - la loi de la conservation de la masse - à son pays, et démantèle l'installation du mythe (littéraire) habsbourgeois juste pour le renouveler à la lumière de la césure nazie ${ }^{40}$.

Outre le vissage sur la composante nazie de la compression ontologique de l'Autriche contemporaine, l'examen attentif de Bernhard se dénoue au travers de l'autre terme qui comprime l'être autrichien : l'immanquable preuve identitaire de la diatribe austro-allemande exposée dans l'incipit de notre discours. La confrontation avec le poids spécifique (historico-culturel) de l'Allemagne se réalise dans le champ esthético-littéraire, notamment par le blâme du "premier homéopathe allemand de l'esprit », Goethe, qui «n'est rien d'autre, au fond, pour les Allemands, qu'un guérisseur [...]. Tout le peuple allemand prend Goethe et s'en trouve bien ", mais son œuvre entière est " la plus grande charlatanerie des Allemands ${ }^{41}$ ». Le " charlatan » Goethe est " le fossoyeur de l'esprit allemand », il est trois fois rien car " c'est Hölderlin qui est le grand poète lyrique [...]. Musil est le grand prosateur et Kleist est le grand auteur dramatique, Goethe, triplement, ne l'est pas $^{42} »$. Toutefois - même en censurant le sommet de la littérature allemande - une "Büroliteratur " petite-bourgeoise, étouffée par les classeurs, le testament poétique de Bernhard capitule à l'" Anschluss tacite » en inscrivant le nom de l'auteur kakanien ${ }^{43}$ par excellence, Musil, dans « la lamentable littérature de fonctionnaires ${ }^{44}$ " (allemande).

Dans les Essais d'herméneutique, Paul Ricour distingue deux pôles dans la constitution de l'imaginaire social : celui de l'idéologie et celui de l'utopie. L'idéologie est le lien imaginaire qui consolide les relations sociales d'une communauté, lorsque l'utopie lui est diamétralement opposée : selon Ricœur, sa fonction est celle de subversion sociale, puisqu'elle met essentiellement la réalité en question. L'imaginaire social repose par conséquent « sur la tension

38 L'expression Austria perennis est utilisée par Hélène Weishard, «De la malédiction d'être autrichien ", in Thomas Bernhard, op. cit.

39 Gerald Stieg, "La satire absolue, Nestroy, Kraus, Bernhard ", in Thomas Bernhard, op. cit., p. 103-106, p. 106.

40 Cf. Stefan Kaszyński, "Die endgültige Zerstörung des Mythos? Zu Thomas Bernhards Theaterstück "Heldenplatz" ", in Österreich und Mitteleuropa: kritische Seitenblicke auf die neuere österreichische Literatur, Poznań, Wydawn. Naukowe UAM, 1995, p. 185-94.

41 Thomas Bernhard, Extinction : un effondrement, op. cit., p. 451-52.

42 Ibid., p. 452.

43 Non sans une certaine ironie, l'écrivain Robert Musil appelait la Double monarchie austrohongroise la "Kakanie ", du préfixe apposé partout K. und K.: Kaiserlich und Königlich (impérial et royal).

44 Ibid., p. 476. 
entre une fonction d'intégration et une fonction de subversion ${ }^{45}$ ". Pourtant, la production d'images en littérature chez Bernhard se place essentiellement dans le champ de l'utopie - elle est une critique acerbe de l'Autriche et des Autrichiens qui, comme le remarque Weishard, en réalité recourt souvent au pronom "nous ", qui a une valeur intégrative. Par exemple, le final du roman Extinction est utopique : le narrateur-protagoniste donne la propriété entière de Wolfsegg à la communauté israélite de Vienne. C'est une utopie radicale et anéantissante qui, plutôt que réfléchir la Hassliebe (haine-amour) de l'auteur pour son pays, s'enracine dans le sentiment de Selbsthass (la haine de soi), dans la malédiction d'être autrichien : même dans son négatif nihiliste, ébréché par des fragments de mythe habsbourgeois, l'Autriche reste l'unique paramètre possible de définition de l'identité de Bernhard.

\section{Sur la malédiction d'être autrichien(ne). Le non-dit}

Si le brisement du silence sur les crimes du passé représente la chute verticale vers le néant de laquelle Bernhard tire son énergie créatrice ${ }^{46}$, dans les écrits d'Ingeborg Bachmann (1926-1973) la comparaison avec la période nazie se concrétise par la mise en question du sens même de l'écriture. Dans le sondage de la société autrichienne, Extinction, en ce sens, se révèle un fil conducteur transtextuel.

Mais si l'écrivaine apparaît plusieurs fois dans l'œuvre de Bernhard ${ }^{47}$, on ne peut pas en dire autant pour ce qui concerne son élaboration de l'héritage nazi. À la différence de Bernhard, Bachmann éprouve une prédilection pour le silence : conformément à la pensée de Ludwig Wittgenstein, elle préfère passer sous silence ce qui ne peut pas être dit, jusqu'aux limites de "l'émanation du désespoir ${ }^{48}$ ". En constatant l'impossibilia de l'expression de la vérité, la nouvelle Trois sentiers vers le lac (Drei Wege zum See, 1972) exemplifie son choix de ne pas se plonger, à l'instar de Bernhard, dans ce qui reste du " moi " autrichien. Vis-à-vis d'une lutte dans la solitude contre les limites du texte ${ }^{49}$, Bachmann essaie ainsi d'ancrer la fragilité de son univers narratif dans le mythe de la Double Monarchie. Dès

45 Paul Ricœur, Le Conflit des interprétations, essais d’herméneutique, Paris, Éd. du Seuil, 1969, p. 391. Cité par Hélène Weishard, "De la malédiction d'être autrichien », in Thomas Bernhard, op. cit., p. 125.

46 Cf. Wendelin Schmidt-Dengler, "Thomas Bernhard, l'Autriche et l'Europe ", in Thomas Bernhard, op. cit., p. 143-150, p. 150.

47 Cf. Bilge Ertugrul, "Thomas Bernhard et Ingeborg Bachmann ", in Thomas Bernhard, op. cit., p. 151-55.

48 C'est une citation de la nouvelle de Bachmann Unter Mördern und Irren (1961), paradigmatique pour illustrer la rupture du silence. Voir : Michel Kappes, "Le motif de l'autodestruction dans la prose de Ingeborg Bachmann ", in Austriaca, n 43, 1996, p. 167-181, p. 170. Voir aussi : Hans Höller, Ingeborg Bachmann: das Werk von den frühesten Gedichten bis zum "Todesarten»-Zyklus, Frankfurt am Main, Athenäum, 1987.

49 Bilge Ertugrul, "Thomas Bernhard et Ingeborg Bachmann », in Thomas Bernhard, op. cit., p. 154. 
le début, la narration commence par un topos de la littérature habsbourgeoise, la gare ferroviaire. Ici, Elisabeth Matrei, la protagoniste, est attendue par son père ; elle revient en sa terre de Carinthie, à Klagenfurt. Sur le chemin du retour aux lieux de l'enfance, ils laissent en taxi la gare et ils tournent dans la Radetzkystrasse ${ }^{50}$. C'est un tournant qui marque la nouvelle entière, échafaudée par renvois continus au monde kakanien ${ }^{51}$ - mythisé par l'écriture de Joseph Roth. Les romans rothiens La Marche de Radetzky (Radetzymarsch, 1932) et La Crypte des capucins (Die Kapuzinergruft, 1938) se poursuivent de cette façon dans les pages de Bachmann. Mais si pour Roth le monde des pages habsbourgeoises était devenu encore plus désirable après la disparition de l'Autriche-Hongrie, parce que la fiction littéraire était plus sûre et rassurante par rapport aux prodromes du nazisme et à l'existence et à la réalité d'apatride, pour Bachmann le propos est différent. Les utopies occidentales sont toujours fortement marquées par la volonté de retrouver un paradis perdu : dans la dimension littéraire, on se leurre à trouver un discours mémorial capable de préserver l'unité trompeuse d'une mémoire fétichiste ${ }^{52}$. Pleinement conscient de cela, la poétique de Roth fait appel à la société habsbourgeoise, en tant que modèle de coexistence ethnique, pour établir un continuum dans la fiction littéraire malgré la disparition de l'Empire austro-hongrois ; la poétique de Bachmann, par contre, s'efforce, en vain, de remplir la discontinuité impossible à combler causée par la fracture nationalsocialiste. Notamment au travers du discours indirect libre, l'Austria perennis est rendue à son anonymat, en sa dilution dans la quotidienneté, en son nivellement généralisant. Bref : en ses idées rę̧ues.

Pour la protagoniste de Trois sentiers vers le lac, le retour à la maison est une occasion pour reparcourir les souvenirs de ses cinquante ans de vie qui l'ont mené loin de sa Carinthie natale : son existence se déroule entre New York et Paris, tout en s'affirmant comme journaliste et photographe, entre un reportage et l'autre qui la conduit au bout du monde. Ses réflexions se développent dans deux directions principales : le désir de retourner au lac, pivot autobiographique du paysage de l'enfance de Bachmann ${ }^{53}$, et la nécessité de faire la clarté sur ses relations sentimentales du passé et du présent.

Le voyage à Klagenfurt de la protagoniste de Trois sentiers vers le lac n'est pas un retour, un vó $\tau \tau o \varsigma$; Elizabeth n'est pas reconduite circulairement chez elle, à son point de départ originaire. Après un bref séjour estival, elle prononce

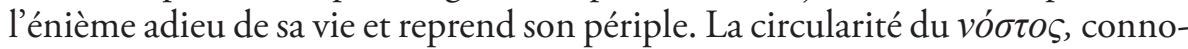
tant un parcours non vain, mais fonctionnel à une acquisition d'expérience et

50 Ingeborg Bachmann, Drei Wege zum See (1972), in Werke, Zweiter Band, München/Zürich, Piper, 1993, p. 395.

51 Voir note 31.

52 Philippe Daros, "Le mythe tel quel ? ", in La Licorne, n 55, 2000, p. 13-27, p. 26.

53 Cf. Hans Höller, "Sur un pays, une rivière et les lacs (Paysage d'enfance) ", in Ingeborg Bachmann, Arles, Actes Sud, 2006, p. 21-28. 
de connaissance, est irréalisable à cause de l'Erfahrungsschwund (W. Benjamin), l'appauvrissement de l'expérience qui, désormais, nous contraint à vivre exclusivement les expériences des autres au travers des médias - aussi l'Extinction de Bernhard prend acte, avec un sentiment d'impuissance, du triomphe du monde photographié, de plus en plus mensonger, mais idéal pour la majeure part de l'humanités ${ }^{\prime 4}$. Dans la nouvelle de Bachmann, il est en somme impossible de constater ce progrès qui n'est relevable et mesurable que par la comparaison avec le point de départ, c'est-à-dire en retournant au lieu dans lequel l'individu peut connaître sa propre identité changée. Toutefois, le texte est

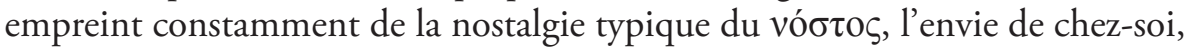
le désir souffert de retourner inhérent à l'étymon grec de la parole nostalgie

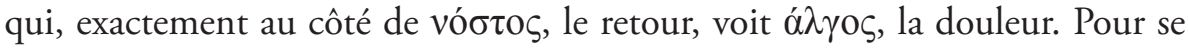
retrouver enfin, Elizabeth revient en Autriche, à sa terre de Carinthie, mais la circularité du retour est irréalisable parce qu'est irréalisable la comparaison avec la réalité du départ, déformée par l'indicible césure nazie et par l'amas de citations kakaniennes ${ }^{55}$. Et si Pietro Citati soutient que Trois sentiers vers le lac est dans une certaine mesure une réconciliation avec la figure paternelle et avec l'Autriche maternelle ${ }^{56}$, Magris croit que l'hommage de Bachmann à la tradition autrichienne est trop explicit ${ }^{57}$. Lécrivaine poursuit la saga du nomade habsbourgeois, en greffant sa mélancolie dissipée sur celle des épigones de 1968 et d'un jet society amorphe et désagrégés ${ }^{58}$, mais son accumulation intertextuelle et les omissisis historiques ne consentent pas à une réconciliation réelle.

\section{Le pays sans qualités}

Sur le non-dit de Bachmann, la mémoire de la Shoah repose silencieuse. À ce propos, Ricœur évoque la singularité d'un phénomène qui met à découvert, "non seulement les limites de la représentation sous ses formes narratives et rhétoriques, mais l'entreprise entière d'écriture de l'Histoire ${ }^{59}$ ". Robert Menasse (né en 1954), un des écrivains autrichiens les plus controversés et discutés de l'époque contemporaine, installe une telle fracture historique dans son œuvre. En particulier, le roman Chassés de l'enfer (Die Vertreibung aus der Hölle, 2001) nous livre une réflexion sur l'histoire, sur ses circonvolutions, sur ses ruptures. Croisant des époques différentes, l'auteur organise le vis-

54 Thomas Bernhard, Auslöschung: ein Zerfall, op. cit, p. 128.

55 Voir note 31.

56 Pietro Citati, Ritratti di donne, Milano, Rizzoli, 1992, p. 285.

57 Claudio Magris, Itaca e oltre, Milano, Garzanti, 1998 (1982), p. 200.

58 Ibid., p. 201.

59 Paul Ricœur, "Devant l'inacceptable: le juge, l'historien, l'écrivain ", in La Philosophie devant la Shoah, "Philosophie ", n 67, Paris, Éd. de Minuit, 2000, p. 14. Cité par Béatrice GonzalésVangell, Kaddish et Renaissance, La Shoah dans les romans viennois de Schindel, Menasse et Rabinovici, Valenciennes, Septentrion, 2005, p. 113. 
à-vis entre ses deux personnages : Manoel Dias Soeiro et Viktor Abravanel, un personnage historique et un personnage de fiction. L'un, Manoel, est un marrane né à Lisbonne le 5 décembre 1604, date d'un monumental autodafé organisé par l'Inquisition; dissimulé sous le prénom chrétien de Manoel, dont le diminutif est "Mané ", l'enfant subit les persécutions d'une société dominée par le fanatisme religieux jusqu'au jour où, avec sa famille, il fuit le Portugal pour la Hollande ; là, à Amsterdam, la "nouvelle Jérusalem ", il retrouvera son identité et deviendra un rabbin célèbre : Manasseh ben Israël - érudit qui fut l'un des maîtres de Baruch Spinoza et probablement aussi l'un des ancêtres de l'auteur du livre. L'autre protagoniste du roman, Viktor, est un historien qui revient sur le passé de sa famille, sur celui de son pays et sur sa propre jeunesse ; né en Autriche au milieu des années 1950, comme Robert Menasse lui-même, Viktor est issu d'une famille mi-juive mi-catholique, dont les origines sont étouffées sous le poids des non-dits. Les deux trames narratives, au lieu d'évoluer parallèlement, se dirigent en réalité l'une vers l'autre ${ }^{60}$. Et dans les dernières pages du roman, Manasseh, désormais proche de sa fin, tente de revenir à " $O$ principio ", l'origine ; il écrit son nom en hébraïque sur une feuille, il la jette. Ses rares souvenirs précoces deviennent littéralement sombres, ils sont tous des souvenirs vespéraux, des images crépusculaires.

L'époque où tout commence, l'enfance, fut pour lui, chose étrange, le soir de la vie ${ }^{61}$.

Pour un des protagonistes de Chassés de l'enfer, le temps du principe est donc le temps de la fin. Si l'Extinction de Bernhard se propose, programmatiquement, d'effacer le temps de l'" infâme enfer provincial "Wolfsegg, pars pro toto de l'Austria perennis sans mémoire, l'écriture de Menasse s'exauce alors, non dans la recherche, peut-être vouée à l'échec, de la vérité historique fondée sur l'événement et le témoignage de "l'enfer ", mais dans la recherche d'une forme littéraire, certes inventée, mais - comme le note Béatrice Gonzalés-Vangel sans doute plus apte à restituer "le continu dans le discontinu ${ }^{62}$ ". (Cela est aussi manifeste dans la nouvelle de Bachmann Trois sentiers vers le lac.)

Cependant, les paradoxes identitaires de l'Autriche reviennent (dans toute leur complexité) dans les écrits de Menasse. Cet auteur d'origine hébraïque est paradigmatique pour illustrer une telle question qu'à son avis, il faudrait analyser en considérant d'abord l'importance assumée par le principe de

60 Béatrice Gonzalés-Vangel, Kaddish et Renaissance, La Shoah dans les romans viennois de Schindel, Menasse et Rabinovici, op. cit., p. 55.

61 Robert Menasse, Chassés de l'enfer (Die Vertreibung aus der Hölle, 2001), trad. par Marianne Rocher-Jacquin et Daniel Rocher, Paris, Verdier, 2005, p. 442.

62 Béatrice Gonzalés-Vangel, Kaddish et Renaissance, La Shoah dans les romans viennois de Schindel, Menasse et Rabinovici, op. cit., p. 304. 
neutralité dans la définition de l'identité autrichienne ${ }^{63}$; dans une Autriche pareille - Das Land ohne Eigenschaften, un pays sans qualités comme le signifie le titre d'un de ses essais - même si la neutralité est une chimère pure, elle éveille dans l'esprit des Autrichiens des associations agréables avec l'idée d'évacuation de toute responsabilité, une évacuation rendue nécessaire par les mauvaises expériences du passé $e^{64}$. Et dans ce cadre de déresponsabilisation de masse, selon Menasse l'intégration européenne a montré et catalysé les déficits identitaires de l'Autriche, en créant (d'ailleurs) les conditions pour l'arrivée au gouvernement d'une personnification de la voix conservatrice du pays : Jörg Haider. Un événement qui, en 2000, à Bruxelles, a mené à la promulgation de sanctions économiques de l'Union européenne contre l'Autriche ; événement qui, en même temps, à Vienne, a conduit à la protestation de dizaines de milliers de personnes rassemblées à l'appel de cinéastes, écrivains, intellectuels. En se démarquant peut-être par un " agnosticisme esthétique ${ }^{65}$ " (à la Kraus) de ses collègues, certes conscient de la force de la provocation destructrice propre de l'œuvre de Bernhard ${ }^{66}$, Menasse - dont une partie de la famille a dû fuir l'Autriche après l'Anschluss - accusa d'opportunisme la mobilisation anti-Haider : "C 'est la minute des hypocrites, l'heure de gloire des idiots qui veulent écrire dans leur notice du whos who : en l'an 2000, j'ai organisé la résistance à $\mathrm{Hitler}^{67}$. "

\section{En guise de conclusion}

À ce point de notre discussion, un propos du germaniste viennois Wendelin Schmidt-Dengler glose magistralement à la fois le non-dit et le dit : la littérature en Autriche mérite à elle seule une recherche approfondie; seul un concept libéré de considérations nationalistes et dégagé de l'approche étroite des histoires traditionnelles de la littérature permet de définir les particularités de la littérature autrichienne car, d'évidence, il n'y a pas de réponse globale à

63 Christian Mariotte, "Les paradoxes identitaires des écrivains autrichiens face à l'intégration européenne : l'exemple de Robert Menasse ", in Problématique de la littérature européenne, Paris, l'Harmattan, 2005, p. 89-96, p. 91.

64 Robert Menasse, Das Land ohne Eigenschaften. Essay zur österreichischen Identität, Wien, Sonderzahl, 1993 (1992), p. 65-80.

65 La définition est de Alfred Pfabigan, Karl Kraus und der Sozialismus: Eine politische Biographie, Vienna, Europa-Verlag, 1976. Cité par Geoffrey C. Howes, "Critical observers of their times: Karl Kraus and Robert Menasse ", in Literature in Vienna at the turn of the centuries: continuities and discontinuities around 1900 and 2000, Rochester, Camden House, 2003, p. 134-51, p. 144.

66 Wendelin Schmidt-Dengler, "Elf Thesen zum Werk Thomas Bernhards ", in Der Übertreibungskünstler: Studien zu Thomas Bernhard, Wien, Sonderzahl, 1986, p. 107-11, p. 107.

67 Robert Menasse, Erklär mir Österreich, Frankfurt am Main, Suhrkamp, 1995, p. 22. Cité par Christian Mariotte, "Les paradoxes identitaires des écrivains autrichiens face à l'intégration européenne : l'exemple de Robert Menasse ", in Problématique de la littérature européenne, op. cit., p. 94. 
la question de la spécificité autrichienne ${ }^{68}$. Cependant, dans cette recherche il faudrait éviter ce que Schmidt-Dengler lui-même remarque dans une autre analyse : la fiction de la continuité autrichienne, la persistance du mythe de l'Empire, qui - déniant les ruptures multiples et insistant sur l'harmonie en tant que fondement de la littérature autrichienne - ramène beaucoup à l'Autriche, surtout à son tourisme Austria felix ${ }^{69}$. D'après Schmidt-Dengler, à l'ombre de cet arbre artificiel s'étale un tableau de l'Autriche qu'il conviendrait de remplacer par un autre, un peu plus conforme à la réalité autrichienne. «Les joyaux ne sont pas exposés au musée ; il faut aujourd'hui fouiller le lit du fleuve de l'histoire de l'Autriche (et par conséquent aussi de Vienne ${ }^{70}$. " Mais, pour aller au-delà du mythe, au-delà du sondage de l'histoire inénarrable, au-delà de la Chanson des Nibelungen et de la ligne de partage rhénan de l'âme allemande, la reconnaissance littéraire/ontologique de l'Autriche devrait en réalité s'aventurer et se perdre dans l'énormité des temps préhistoriques, dans ce que Magris définit comme le "fracas d'une gigantesque mise en place ", la " géographie de Titans " dans laquelle l'Urdonau, le Danube primitif qui recevait les eaux de l'Ur-Rhin, "vers la moitié du Tertiaire, pendant l'Eocène, il y a vingt à soixante millions d'années, avait son embouchure à peu près sur le site actuel de Vienne, dans un golfe de Thétis, mère originelle des océans, au bord de cette mer des Sarmates qui recouvrait alors toute l'Europe du sud-est ${ }^{71}$ ».

\title{
Igor Fiatti
}

Paris 3 - La Sorbonne Nouvelle Università degli Studi di Torino igorfiatti@gmail.com

\begin{abstract}
Résumé
L'Autriche et sa production littéraire semblent condamnées à un désarroi existentiel, à tout jamais. Dans une telle recherche continuelle de lueur d'indices et de preuves identitaires, la résistance opposée aux engrenages de la puissante machine critico-éditoriale de l'" Anschluss tacite " est constituée, notamment, par des fragments de mythe, qui, mutatis mutandis, renvoient aux sécurités de l'époque de l'ordo habsbourgeois.
\end{abstract}

\section{Mots-clés}

Autriche, Allemagne, mythe habsbourgeois, identité, Danube.

68 Cf. Wendelin Schmidt-Dengler, Bruchlinien. Vorlesungen zur österreichischen Literatur 1945 bis 1990, Wien, Residenz, 1995.

69 Wendelin Schmidt-Dengler, "Vienne 1918 : finale sans éclat ", in Continuités et ruptures dans la littérature autrichienne : 17 essais, op. cit., p. 175-198, p. 196.

70 Id.

71 Claudio Magris, Danube, op. cit., p. 24. 


\section{Abstract}

Compressed between the historic-cultural specific weight of Germany and the immanence of the Nazi past on the collective consciousness, Austria and its literary production seem condemned to a perpetual existential confusion. In such a continuous research of identity's proofs, the resistance opposed to the "tacit Anschluss " and its critique-editorial machine is mostly constituted by fragments of myth that, mutatis mutandis, refer to the security of the habsburgic time ordo.

\section{Keywords}

Austria, Germany, Habsburg myth, identity, Danube. 